\section{The Mouth Care Matters programme}

\author{
Kate Quinlan visited East Surrey Hospital to learn more about Mouth Care \\ Matters, an initiative with patient health and dignity at its heart.
}

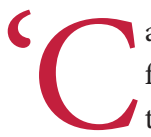
an you imagine how it would feel if you hadn't brushed your teeth for a week, or if your mouth became so dry through dehydration that your tongue became stuck to your palate?' For Mili Doshi, Consultant in Special Care Dentistry in the Dental \& Maxillofacial Department at East Surrey Hospital, putting the 'mouth back in the body' for hospital inpatients has been top priority since 2015, when she became responsible for developing the Health Education England (HEE) Mouth Care Matters programme in hospital.

'We needed to raise awareness amongst staff and patients of the importance of oral health', explains Mili. 'The oral cavity is the gateway to our body; we need a functioning mouth to eat, speak, and smile. If we don't remove the bacteria in our mouths by daily brushing, it can lead to aspiration pneumonia, a condition with high mortality rates extending hospital stay by an average of eight days. There is evidence that poor oral health is linked to systemic conditions such as aspiration pneumonia, diabetes, cardiovascular disease and even dementia.

I travelled to East Surrey Hospital in May to learn more about Mouth Care Matters and meet the Leads who are currently implementing the programme across 12 hospital trusts in Kent, Surrey and Sussex. The 17 Mouth Care Matters Leads are all seconded to the 12-month programme; nine are dental nurses, two are speech and language therapists, and six are registered nurses. On 2 May they were attending their sixth study day as part of the programme.

According to Mili, the poor state of mouth care for hospital patients is not the fault of hospital staff. 'Sometimes it can be as simple as not having a stock of toothbrushes on a ward, and nursing assistants, who attend to most mouth care, often have no training.
How can we expect someone to support tooth brushing for a patient with dementia who is saying "no, no, no", if they haven't had training on how to manage this? Training staff so they understand why oral health is so important and arming them with the skills needed to deliver it is therefore vital. Hospitals must also be adequately stocked with appropriate mouth care products.'

\section{Mouth Care Matters}

East Surrey Hospital, run by Surrey and Sussex Healthcare NHS Trust, is the first in the country to have a specialist mouth care team of dental care professionals to improve the oral health of hospital inpatients. The Mouth Care Matters initiative provides training to nursing staff, doctors and allied healthcare professionals so that patients admitted for more than 24 hours receive good mouth care during their stay at East Surrey Hospital.

There are four key drivers for the programme:

- Being in hospital has an impact on oral health

- We have an ageing population, so there are more older people who need care

- Our mouths are changing, with more people keeping their teeth

- We are taking more medication, and dry mouth is a common side effect of over 40 medicines.

The Mouth Care Matters initiative has seen a rise in the number of referrals to the on-site dental department and mouth care team and has benefited patients in a range of ways - from making mouths more comfortable, helping patients to eat and drink and correctly treating oral infections - to support for palliative care patients with sore ulcerated mouths.

\section{Developing MCM}

Jenny Chay, a dental nurse and oral health promoter at East Surrey Hospital, explained some of the background to the Mouth Care Matters programme.

'Health Education Kent, Surrey and Sussex [HEKSS] were already running a programme called IOHOPI: the Improving the Oral Health of Older People Initiative. They asked Surrey and Sussex Health Care Trust to develop a branch on the programme focussing on patients in hospital.'

In September 2015 Mili Doshi was approached to run the project, and with Jenny's help they set out to take a closer look at the oral health of inpatients in East Surrey Hospital.

'Just before we were asked to do the project,' says Jenny, 'the Care Quality Commission [CQC] had come into the hospital and their only clinical finding was that the patients had very dry mouths and there was no consensus of opinion over where you should record mouth care. So one of the CQC's recommendations was that we implement a tool to address that point.

'That was a really good starting point for us. We started with the very basics, going and having a look at what we had in the hospital, what tools we were using, where things were being recorded, and what the state of affairs was.

'The hospital had so many examples of wonderful care but mouth care did need to improve. When we started this initiative we came across one older patient who was waiting to be discharged who developed a very dry mouth and started to bite her lip constantly. She stopped eating and drinking, developed hospital acquired pneumonia and almost died. We showed the staff how to care for her dry mouth and treat the lip ulceration and the patient recovered. Sometimes it is the little things.'

HEE secured further funding so that a further 12 trusts could fund a mouth care lead for 12 months.

'We started putting a training programme together and consequently we advertised and we've now got 12 trusts with a Mouth Care Matters Lead,' explains Jenny. 'They train with us and return for study days and we've got three different cohorts. They are all working hard and we are learning from each other and further developing the programme.' 


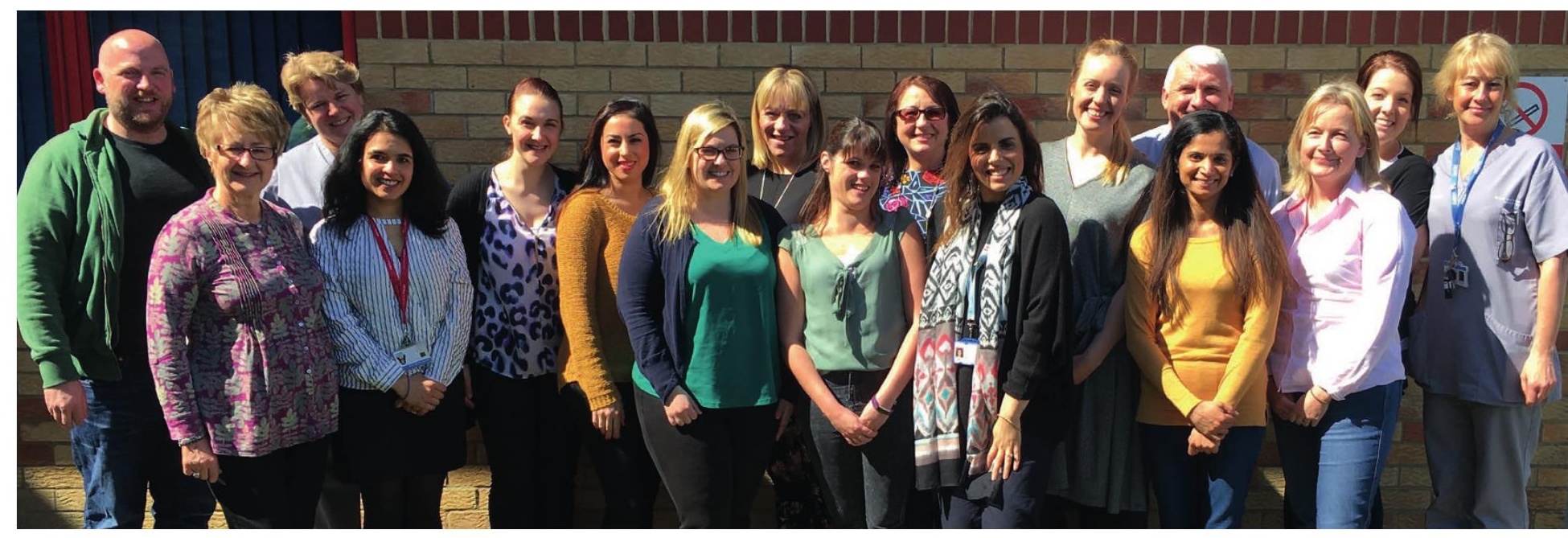

Mouth Care Matters Leads from 12 trusts across Kent Surrey and Sussex, with Jenny Chay (third from the left) and Mili Doshi (fourth from the left)

\section{Training hospital staff}

The Leads' role is primarily to train hospital staff in mouth care rather than to provide mouth care themselves. Mouth care training is now part of the mandatory training that all staff at East Surrey Hospital have to undertake annually. The Mouth Care Leads have also provided training programmes for nursing assistants, doctors, physiotherapists, occupational therapists, speech and language therapists, the diabetic team, radiologists, and even outpatients staff.

'The more people in the hospital we can raise awareness in, says Jenny, 'the more likely it is that mouth care will be carried out.'

As part of the initiative, a mouth care screening sheet is completed for all patients 24 hours after admission. The screening sheet indicates whether a patient is independent - can walk to a sink, stand and brush their teeth, and has the equipment to do so; requires some assistance (they may need a bowl, encouragement or a reminder, for example); or is fully dependent on others for mouth care, in which case they require an assessment by the dental team on-site.

\section{Dental products}

The Mouth Care Matters programme has also led to a change in the products available in the hospital, so that the same products are available on every ward: a toothbrush, toothpaste and a dry mouth gel. Jenny explains: 'We now have a small, soft-headed toothbrush that's available from NHS Supply Chain and available on the wards. The toothbrush used to look like a nailbrush, with a big hard head and a short stubby handle. If you look at vulnerable, frail patients, it was very difficult to brush their teeth with those.
'We also have a non-foaming (SLS free) toothpaste that can be used for all patients. What we didn't want was nursing staff to keep making lots of decisions. That's when mistakes are made. The dry mouth gel is not on prescription so it can be handed out to people to put on themselves or we can support patients to use it.'

The programme has also introduced a sunflower sticker which can be used to indicate that a patient may have a denture. Dentures commonly go missing in hospitals in pillowcases, dressing gown pockets and on dinner trays. Auxiliary staff, porters and housekeepers have been trained to look out for dentures when they see a sunflower sticker. Denture pots with lids have also been introduced.

\section{Spreading the word}

East Surrey dental core trainee Jessica Mann has been helping Mili Doshi to spread the word about mouth care. On 28 April Jessica and Mili spoke at the British Geriatrics Society's Spring Meeting in Gateshead, helping to raise awareness of the importance of oral health among medical specialties who may not have considered it. Says Mili: 'Most doctors receive no oral health training in their undergraduate or postgraduate training and so it is perhaps unsurprising that in a recent hospital-based survey, $100 \%$ of junior doctors said they did not feel very confident in diagnosing oral-related conditions. Training in diagnosis and management of common oral conditions such as oral thrush and mucosal ulceration is essential. Incidence rates for oral cancer are projected to rise by $33 \%$ in the UK by 2035 (to 20 cases per 100,000 people) - doctors must therefore be aware of the early signs and symptoms if better outcomes are to be achieved'.

The Mouth Care Matters programme will also be holding a conference on 7 July 2017 called 'Mouth Care Matters - can you afford not to?' This is designed to teach delegates about the underpinning reasons for the Mouth Care Matters programme and encourage other trusts to support Mouth Care Leads in their hospitals.

Mili hopes that the programme will expand to other areas of the country: 'the initiative is relevant for all people who provide personal care to patients be that in an acute, care home or community setting. Oral health is an important part of general health and wellbeing. It allows people to eat, speak, smile and also socialise without discomfort or embarrassment. Poor oral health can lead to deterioration in general health. This links to quality of life and dignity'.

From Jenny's point of view, oral health has always been important, but has slipped off the agenda: 'When I was young I used to have my temperature taken in my mouth. Hospital thermometers were the ones you put into the mouth so somebody would have looked in patients' mouths three or four times a day. Now we wave something over their ear and a problem in the mouth can disappear by the patient pushing their lips together. It has just been forgotten that if a patient can't eat, the first thing that you should look at is the first place the food goes: the mouth. We've had cases where the denture's been left at home and the patient's been given food but they can't chew it, or their mouth is so dry they can't open the mouth to get the food in ... so we start with the very basics. That's why I think the MCM programme is so successful, because it's so simple'.

The full version of this article was originally published in BDJ Team on 2 June 2017: http://go.nature.com/2r4XXA2. 